\title{
The Future of Pediatric Hospital Medicine: Challenges and Opportunities
}

\author{
Marie E Wang, MD, MPH, Erin E Shaughnessy, MD, MSHCM², JoAnna K Leyenaar, MD, PhD, MPH³
}

'Division of Pediatric Hospital Medicine, Stanford University School of Medicine, and Lucile Packard Children's Hospital Stanford, Stanford, California; ${ }^{2}$ Division of Hospital Medicine, Phoenix Children's Hospital, and Department of Child Health, University of Arizona College of Medicine-Phoenix, Phoenix, Arizona; ${ }^{3}$ Department of Pediatrics and the Dartmouth Institute for Health Policy \& Clinical Practice, Dartmouth-Hitchcock Medical Center, Lebanon, New Hampshire.

ediatric hospital medicine (PHM) is in the midst of an exciting period of growth. In 2016, the American Board of Medical Specialties approved the petition for PHM to become the newest pediatric subspecialty, taking PHM on a divergent path from the Focused Practice in Hospital Medicine designation established for adult hospitalists. Establishment as a subspecialty has allowed PHM to define the unique skills and qualifications that hospitalists bring to patients and the healthcare system. These skills and qualifications are delineated in the PHM core competencies and national fellowship curriculum..$^{1,2}$ In order to realize the vision of PHM to improve care for hospitalized children described by Roberts et al., ${ }^{3}$ concerted efforts are needed to train and retain a workforce that is equipped with the skills to catalyze improvements in inpatient pediatric care. We discuss challenges and opportunities facing PHM in workforce development, sustainability of clinical work models, and interhospital collaboration.

\section{FELLOWSHIP TRAINING AND \\ THE PHM PIPELINE}

The development of PHM as a subspecialty was driven by a number of factors. ${ }^{4}$ The acuity of hospitalized children has increased significantly, with a population comprised of more children with complex chronic conditions and/or technology dependence, serious complications of acute conditions, and acute mental health problems. At the same time, the medical and behavioral conditions seen by outpatient general pediatricians have become more complex and time intensive, with these practitioners less likely to work in inpatient settings. Hospitalist care has positive impacts on healthcare efficiency and value, and both parents and primary care pediatricians report high levels of satisfaction with the healthcare delivered by PHM services. ${ }^{4}$

A national count of the number of pediatric hospitalists is currently lacking. Conservative estimates suggest that at least 3,000 pediatric hospitalists currently practice in the United States. ${ }^{5}$ These hospitalists have highly varied scopes of prac-

\footnotetext{
*Corresponding Author: Marie E. Wang, MD, MPH; Email: marie.wang@
} stanford.edu; Telephone: (650) 736-4423.

Published online first February 19, 2020.

Received: November 15, 2019; Revised: December 11, 2019;

Accepted: December 13, 2019

๑ 2020 Society of Hospital Medicine DOI 10.12788/jhm.3373 tice and work across diverse settings-more diverse, perhaps, than any other pediatric subspecialty. Although difficult to quantify, we estimate that approximately one-third of pediatric hospitalists in the US work in community hospitals and the remainder practice at children's hospitals. ${ }^{6}$ Many of the needs of hospitalized children differ across these settings, and the roles and challenges faced by hospitalists in these settings correspondingly differ. Community hospitalists frequently take active roles in newborn care and emergency department consultation, often without the support of other pediatric subspecialties. ${ }^{7}$ In contrast, hospitalists working at children's hospitals more frequently care for highly complex patients, often collaborate across multiple specialties and assume nonclinical roles in quality improvement (QI), research, and medical education.

Residents graduating in July 2019 were the last cohort of residents eligible to pursue PHM subspecialty certification via the practice pathway. Accordingly, future residency graduates interested in PHM subspecialty certification will need to complete a PHM fellowship at an accredited program in the US or Canada. Since 2008, PHM fellowship directors have met yearly to collaborate and share best practices, ${ }^{8}$ developing the twoyear fellowship curriculum that forms the basis for the American Board of Pediatrics training pathway. ${ }^{2}$ The curriculum allows significant flexibility to meet diverse needs, including tailored content for fellows planning to practice in community settings, fellows planning research careers, medicine-pediatrics hospitalist careers, and those desiring increased training in $\mathrm{Ql}$, medical education, or leadership/administration. ${ }^{2}$ In the spring of 2019, Pediatric Research in Inpatient Settings (PRIS) leadership, directors of existing PHM fellowship programs, and national academic society representatives met to develop a fellows' research curriculum, training resources, and guidelines around scholarship expectations. ${ }^{9}$ This collaboration aims to accelerate the growth of high-quality clinical training and scholarship to benefit hospitalized children across many different settings.

Such collaboration is essential to address an emerging workforce challenge in PHM. Although the number of PHM fellowship positions is expected to grow in the coming years, there is currently a shortage relative to the anticipated demand. With approximately 2,800 US pediatric residents graduating annually and data indicating that $7 \%$ of graduating residents enter and remain in PHM for at least five years, ${ }^{10,11}$ almost 200 fellowship spots may be needed each year. As of November 2019, 77 fellowship positions were available for residents graduating in 2020, ${ }^{12}$ which is less than half of the potential demand. To 
address this mismatch, the PHM Fellowship Directors' Council has led an annual training for new and potential fellowship directors, and 18 new programs are under development. ${ }^{13}$ However, this growth may be inadequate to meet the needs of the field. The extent to which limited PHM fellowship positions will adversely affect the pipeline of pediatricians pursuing PHM is unknown.

Efforts to support institutions in creating and expanding fellowship programs will be needed to address the potential shortage of fellowship positions. Continued guidance from the PHM Fellowship Directors' Council in the many aspects of fellowship program development (eg, curriculum design, assessment) will be crucial in this endeavor. Furthermore, given that fellowships must support fellows to conduct scholarly work and demonstrate evidence of robust faculty scholarly activities to attain accreditation, an essential area of focus is faculty development. Considering barriers such as lack of time, mentorship, and resources, some divisions interested in starting a fellowship may find it challenging to achieve these standards. ${ }^{14}$ However, hospitalists are often engaged in areas such as QI and medical education, and there is potential to turn ongoing work into meaningful scholarship with appropriate guidance. Many of our supporting organizations (eg, Academic Pediatric Association, American Academy of Pediatrics, and Society of Hospital Medicine) provide training programs for faculty in areas such as educational scholarship, research, and Ql; however, more may be needed. Leaders of PHM programs will need to be mindful and creative in accessing local, regional, and national resources to invest in faculty development.

\section{CLINICAL WORK MODELS AND SUSTAINABILITY}

As a group, pediatric hospitalists report high levels of satisfaction with their jobs. ${ }^{11}$ Despite this finding, there are a number of threats to the sustainability of current work models, some of which are unique to pediatrics given the overall lower patient volumes and greater seasonal variation compared with adult hospital medicine. Both university and community-based hospitalist programs report high weekend, overnight, and in-house clinical effort. ${ }^{7,15}$ Recent studies reported that a significant proportion of PHM program leaders (50\% of division directors at university-affiliated programs and $37 \%$ of community program leaders) perceive their program to be unsustainable. ${ }^{7,15}$ Among university-affiliated programs, a higher burden of weekend work as well as university employment were associated with perceived unsustainability, while no specific program or employer characteristic was associated with this perception in community programs.

These findings indicate that efforts are needed to address PHM program sustainability and that different work models and interventions may be needed for university-based and community PHM programs. Wide variability exists in the ways that programs address overall clinical burden, with strategies including census caps, seasonal expansion of coverage, and formal back-up systems. ${ }^{715}$ Additional potential solutions may include differential weighting or financial incentives for nights and weekends, support for nonclinical work, loan repayment programs, and competitive salaries. ${ }^{11}$ In addition, structuring clinical and nonclinical roles to facilitate career development and advancement may enhance career longevity. ${ }^{15}$ Lessons learned from pediatric emergency medicine (PEM), which developed as a field a few decades ahead of PHM, may predict future challenges. A 2015 survey of PEM faculty found that despite a $15 \%$ decrease in weekly work hours over a 15-year period, a substantial number of PEM faculty report concerns about burnout, with $40 \%$ reporting a plan to decrease their clinical workload and $13 \%$ planning to leave the field within five years. ${ }^{16}$ Like PEM, the field of PHM may benefit from the development of best practice guidelines to improve well-being and career longevity. ${ }^{17}$

\section{INTERHOSPITAL COLLABORATION}

The culture of collaboration within PHM places the field in a solid position to address both workforce challenges and barriers to high-quality care for hospitalized children. There are several hospital-based learning networks actively working to strengthen our knowledge base and improve healthcare quality. The PRIS network (www.prisnetwork.org) aims to improve healthcare for children through multihospital studies, boasting 114 sites in the US and Canada. Numerous collaborative projects have linked hospitalists across programs to tackle problems ranging from handoff communication ${ }^{18}$ to eliminating monitor overuse. ${ }^{19}$ The Value in Inpatient Pediatrics network has similarly leveraged collaborations across multiple children's and community hospitals to improve transitions of care ${ }^{20}$ and care for common conditions such as bronchiolitis, febrile infants, and asthma. ${ }^{21}$ These networks serve as models of effective collaboration between children's hospitals and community hospitals, more of which is needed to increase research and Ol initiatives in community hospitals, where the majority of US children receive their hospital-based care., 622

With the rapid growth of scholarly networks in research, $\mathrm{Ql}$, and education, PHM has a solid infrastructure on which to base continued development as a subspeciality. Building on this infrastructure will be essential in order to address current challenges in workforce development, fellowship training, and program sustainability. Ultimately, achieving a strong, stable, and skilled workforce will enable PHM to fulfill its promise of improving the care of children across the diversity of settings where they receive their hospital-based care.

Disclosures: Dr. Leyenaar provides consultative services to the American Board of Pediatrics Foundation, which is not associated with this manuscript. Drs Wang and Shaughnessy have no disclosures.

\section{References}

1. Stucky ER, Maniscalco J, Ottolini MC, et al. The Pediatric Hospital Medicine Core Competencies Supplement: a framework for curriculum development by the Society of Hospital Medicine with acknowledgement to pediatric hospitalists from the American Academy of Pediatrics and the Academic Pediatric Association. J Hosp Med. 2010;5(S2):1-114. https://doi.org/10.1002/ jhm.776

2. Jerardi $K E$, Fisher $E$, Rassbach $C$, et al. Development of a curricular frame- 
work for pediatric hospital medicine fellowships. Pediatrics. 2017;140(1) https://doi.org/10.1542/peds.2017-0698

3. Roberts KB, Fisher ER, Rauch DA. The history of pediatric hospital medicine in the United States, 1996-2019. J Hosp Med. 2020;15(7):424-427. https://doi. org/10.12788/jhm.3381

4. Barrett DJ, McGuinness GA, Cunha CA, et al. Pediatric hospital medicine: A proposed new subspecialty. Pediatrics. 2017;139(3). https://doi.org/10.1542/ peds.2016-1823

5. American Board of Medical Specialities. American Board of Medical Specialities application for a new subspecialty certificate: Pediatric hospital medicine. Accessed November 6, 2019. http://www.abms.org/media/114649/ abpeds-application-for-pediatric-hospital-medicine.pdf

6. Leyenaar JK, Ralston SL, Shieh MS, et al. Epidemiology of pediatric hospitalizations at general hospitals and freestanding children's hospitals in the United States. J Hosp Med. 2016;11(11):743-749. https://doi.org/10.1002 /jhm.2624

7. Alvarez F, McDaniel CE, Birnie K, et al. Community pediatric hospitalist workload: results from a national survey. J Hosp Med. 2019;14(10):682-685. https://doi.org/10.12788/jhm.3263

8. Shah NH, Rhim HJ, Maniscalco J, Wilson K, Rassbach C. The current state of pediatric hospital medicine fellowships: A survey of program directors. J Hosp Med. 2016;11(5):324-328. https://doi.org/10.1002/jhm.2571

9. Pediatric Hospital Medicine Fellowship Research Training Development. Accessed December 10, 2019. https://projectreporter.nih.gov/project_info_description.. $\mathrm{fm}$ ?aid $=9593276 \&$ icde $=47889643$

10. Leyenaar JK, Frintner MP. Graduating pediatric residents entering the hospital medicine workforce, 2006-2015. Acad Pediatr. 2018;18(2):200-207. https:// doi.org/10.1016/j.acap.2017.05.001

11. House S, Frintner MP, Leyenaar JK. Factors influencing career longevity in pediatric hospital medicine. Hosp Pediatr. 2019;9(12):983-988. https://doi. org/10.1542/hpeds.2019-0151
12. PHM Fellowship Programs. Accessed November 6, 2019. http://phmfellows.org/phm-programs/

13. Rassbach $C$ [Personal communication]; 2019

14. Bekmezian A, Teufel RJ, 2nd, Wilson KM. Research needs of pediatric hospitalists. Hosp Pediatr. 2011;1(1):38-44. https://doi.org/10.1542/ hpeds.2011-0006

15. Fromme HB, Chen CO, Fine BR, Gosdin C, Shaughnessy EE. Pediatric hospitalist workload and sustainability in university-based programs: Results from a national interview-based survey. J Hosp Med. 2018;13(10):702-705. https:// doi.org/10.12788/jhm.2977

16. Gorelick MH, Schremmer R, Ruch-Ross H, Radabaugh C, Selbst S. Current workforce characteristics and burnout in pediatric emergency medicine. Acad Emerg Med. 2016;23(1):48-54. https://doi.org/10.1111/acem.12845

17. American College of Emergency Physicians. Policy Statement: Emergency Physician Shift Work; June 2017

18. Starmer AJ, Spector ND, Srivastava R, et al. Changes in medical errors after implementation of a handoff program. N Engl J Med. 2014;371(19):18031812. https://doi.org/10.1056/NEJMsa1405556

19. Rasooly IR, Beidas RS, Wolk CB, et al. Measuring overuse of continuous pulse oximetry in bronchiolitis and developing strategies for large-scale deimplementation: Study protocol for a feasibility trial. Pilot Feasibility Stud. 2019;5:68. https://doi.org/10.1186/s40814-019-0453-2

20. Coghlin DT, Leyenaar JK, Shen M, et al. Pediatric discharge content: a multisite assessment of physician preferences and experiences. Hosp Pediatr. 2014;4(1):9-15. https://doi.org/10.1542/hpeds.2013-0022

21. Value in inpatient pediatrics (VIP) Network. 2019. Accessed October 13, 2019. https://www.aap.org/en-us/professional-resources/quality-improvement/ Pages/Value-in-Inpatient-Pediatrics.aspx

22. McDaniel $C E$, Jennings $R$, Schroeder $A R$, et al. Aligning inpatient pediatric research with settings of care: a call to action. Pediatrics. 2019;143(5). https:// doi.org/10.1542/peds.2018-2648 